\title{
Non-conserving Flow Aspect of Maximum Dynamic Flow Problem
}

\author{
Phanindra Prasad Bhandari, Shree Ram Khadka \\ Central Department of Mathematics, Tribhuvan University, Kathmandu, Nepal \\ Corresponding author:phanindra.maths@gmail.com
}

Received: Dec 12, 2017

Revised: Jan 15, 2018

Accepted: Jan 25, 2018

\begin{abstract}
Shifting as many people as possible from disastrous area to safer area in a minimum time period in an efficient way is an evacuation planning problem (EPP). Modeling the evacuation scenarios reflecting the real world characteristics and investigation of an efficient solution to them have become a crucial due to rapidly increasing number of natural as well as human created disasters. EPPs modeled on network have been extensively studied and the various efficient solution procedures have been established where the flow function satisfies the flow conservation at each intermediate node. Besides this, the network flow problem in which flow may not be conserved at nodes necessarily could also be useful to model the evacuation planning problem. This paper proposes an efficient solution procedure for maximum flow evacuation planning problem of later kind on a single-source-single-sink dynamic network with integral arc capacities with holding capability of flow (evacuees) in the temporary shelter at intermediate nodes.
\end{abstract}

Keywords: EPP, dynamic network flow, minimum cost flow problem, preflowpush algorithm

\section{Introduction}

Potential increment in natural and human created disasters that cause massive destruction of properties including the human casualties have drawn the attention of researchers to find efficient emergency management procedures so that the casualties and the destructions could be reduced. Some worth-mentioning disasters are recent (2015) earthquake in Nepal, Chichi Bam and Kashmir earthquakes in Taiwan, Iran and Pakistan, the tsunami in Indian Ocean and Japan, Fukushima nuclear accident in 2011, September-11 attack in the USA, and hurricanes like Rita and Katrina in 2005. Besides, it is useful for the management of mass-meetings and to mitigate the traffic situation in busy traffic hours. Evacuation planning is a part of overall emergency management that includes prevention, planning, response and recovery (PPRR). 
An evacuation planning problem attempts to find an optimal evacuation plan in a realistic flow model where each evacuee is supposed to be evacuated in an efficient way in given time period. We take the network flow optimization approach to deal the evacuation planning problem. An evacuation network consists of the source (dangerous place), the sink (safe place), the intermediate nodes and the arcs (road-segments between any two nodes) with some given capacities. The intermediate nodes serve with temporary shelters with sufficient capacities besides serving as transshipment nodes. The source contains evacuees and the sink waits them with sufficient capacities. The movement of the evacuees in the path-segment is the flow in the arc.

Ford and Fulkerson [7] were the first authors who introduced network flow problem that sends the maximum amount of flow from the source to the sink known as maximum flow problem where the flow function satisfies the conservation constraints at each intermediate node. Their solution to the problem is based on arbitrary source-sink path augmentation on the residual networks and runs only in pseudo polynomial time. Edmonds and Karp [6] choose a shortest source-sink path in the residual network in each iteration for augmentation that improves the running time of the algorithm to polynomial time. The idea of augmenting all the shortest paths at once in each iteration in a layered sub-network of the residual network is given by Dinic [5]. This further improves the efficiency of the algorithm in [6].

The variety of maximum evacuation flow problems with different flavors have been studied with flow conservation aspects. The flow problem that maximizes the flow from a source to a sink in given time horizon is a maximum dynamic flow problem. Ford and Fulkerson $[8,9]$ showed that the general maximum dynamic flow problem is equivalent to the static flow problem in the timeexpanded network and solved the problem in two-terminal network by temporarily repeating the static solution in the given network itself considering the transit times of the arcs as cost coefficients. The quickest flow problem minimizes the total time to send the given flow from the source to the sink. Solution to the problem with two terminals and multi terminals can be found in Burkard et al. [3] and Hoppe [12] respectively. The earliest arrival flow problems that ask to maximize flow into the sink at each time step within the time horizon have been extensively studied in the literature; Gale [10], Minieka [17], Wilkinson [24], Hoppe [12], Hoppe and Tardos [13], Baumann [1], Steiner [23] and Ruzika et al. [22]. The evacuation planning problem with another flavor is contraflow approach. Transformation of network by reversing the direction of the arc into the ideal direction and reallocating the available capacity for maximizing the flow and minimizing the evacuation time from source to sink is the network contraflow evacuation planning problem. Rebennack et al. [21] studied the maximum static contraflow problem for general network, the maximum dynamic contraflow problem and the quickest contraflow problem for two terminal networks and presented the polynomial time algorithms but the quickest transshipment contraflow problem and fixed switching cost contraflow problems are shown NP-hard. Extensive studies with contraflow approach have been made by the authors Dhamala and Pyakurel [4], Pyakurel et al. [20], Pyakurel and Dhamala [18], Khadka and Bhandari [15] and Pyakurel and Dhamala [19]. 
The concept of preflow, pushing as much flow as possible out of the source and trying to get it to the sink without satisfying the flow conservation by the flow function at nodes necessarily, was first introduced by Karzanov [14]. Goldberg and Tarjan [11] showed this concept as an efficient tool to solve the maximum flow problem in network and gave the efficient algorithm known as preflow-push algorithm. This approach sends the excess flow (evacuees) that is blocked to send into the sink back to the source and deletes from entire evacuation network since the flow conservation property is not maintained at nodes. The algorithm has been modified by Khadka and Bhandari [16] and Bhandari and Khadka [2] to solve the maximum evacuation planning problem on the network with holding capability of the flow on the temporary shelter of the intermediate nodes. They proposed a relaxation to allow holding evacuees in the temporary shelter at intermediate nodes instead of sending them back to the source. This relaxation could be more advantageous in many ways. A detail can be found in [16] and [2].

The paper is organized as follows: In Section 2, we formulate the maximum dynamic evacuation planning problem with holding capability of evacuees in temporary shelter at intermediate nodes based on non-conserving flow model. The preflow-push algorithm and its modification have been presented in Section 3. A new and efficient solution procedure to the problem is proposed in Section 4, and finally Section 5 concludes the paper.

\section{Problem Formulation}

An evacuation scenario is modeled as a network $N=(V, E)$ where $E(|E|=m)$ is the set of road segments termed as arcs and $V(|V|=n)$ is for the set of the intersections of the arcs termed as nodes such that $m \geq n-1$. Number of lanes on the road has been taken as the capacity $c_{e} \geq 0$ and the time taken to travel from a node $v$ to $w$ is the transit time $\tau_{e}$ for each arc $e=(v, w) \in E$. Unsafe place where disaster has occurred or going to be occurred soon is taken as the source $s$ and the safe place where the people are to be evacuated is assumed as the sink $d$. The source contains evacuees, and the sink waits them for shelter with enough space. We assume the temporary shelter $v^{\prime}$ at every node $v \in V-\{s, d\}$ with sufficient capacity of $c_{v}$. Total time horizon within which evacuees are to be shifted to the safe place is $T$. The movement of evacuees on the road is considered as the flow on the network $N$.

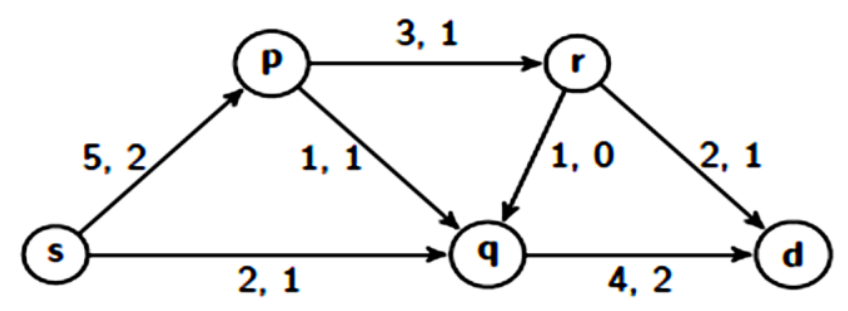

Fig. 1: An evacuation network $N$ with $s$ and $d$ as the source and the sink respectively. The first and the second numbers next to each arc are the capacity and the transit time respectively

The preflow $f$ on the dynamic network $N=\left(V, E, c_{e}, \tau_{e}, s, d, T\right)$ is an assignment $f: E \times$ $\{0,1, \ldots, T\} \rightarrow R^{+} \cup\{0\}$ satisfying the following constraints. 
The capacity constraints

$$
f(e, \theta) \leq c_{e} \forall e \in E \text { and } \forall \theta \in\{0,1, \ldots, T\} .
$$

The skew symmetry of the flow at each time step $\theta \in\{0,1, \ldots, T\}$ along the arc as

$$
f(v, w)=-f(w, v) \quad \forall v, w \in V .
$$

And the constraint with no flow conservation as

$$
\sum_{e \in \delta^{+}(v)} \sum_{\theta=0}^{T} f(e, \theta) \geq \sum_{e \in \delta^{-}(v)} \sum_{\theta=0}^{T} f(e, \theta)
$$

where $e \in \delta^{+}(v)$ and $e \in \delta^{-}(v)$ denote the arcs entering to and leaving from the node $v$ respectively.

We consider a non-negative excess at node $v$ defined as

$$
e_{v}=\sum_{w \in V} f(w, v)
$$

and call the node $v$ an active node if $e_{v}>0$. We define the nonnegative overload at node $v$ as

$$
o_{v}=\sum_{w \in V} f(w, v)-\sum_{w \in V} f(v, w) \geq 0 \forall v \in V-\{s, d\}
$$

satisfying

$$
o_{v} \leq c_{v}, \quad \forall v \in V-\{s, d\}
$$

The overload flow at every node $v \neq s, d$ is allowed to hold at the temporary shelter $v^{\prime}$ of the node $v$ for each time step $\theta \in\{0,1, \ldots, T\}$.

The objective of the maximum dynamic flow problem on the network $N$ is to maximize the flow

$$
f_{d}=\sum_{e \in \delta^{+}(d)} \sum_{\theta=0}^{T} f(e, \theta) \text {. }
$$

\section{The Preflow-Push Algorithm and its Modification}

The preflow-push algorithm of Goldberg and Tarjan [11] is an iterative procedure that updates the preflow in the residual network in each iteration. For the network $N=\left(V, E, c_{e}\right)$, a residual network $N_{f}=\left(V, E_{f}, c_{e}\right)$ is constructed with respect to the preflow $f$. For an arc $e=(v, w)$ with capacity $c_{e}$ and carrying preflow $f(e), N_{f}$ includes arcs in forward direction of arc $e$ with residual capacity $r_{e}=c_{e}-f(e)$ and arcs in reverse direction of arc $e$ with residual capacity $r_{e}=f(e)$. The algorithm starts with the initialization of the given network $N$. At the beginning, the preflow $f$ on each arc leaving the source is equal to the arc capacity of the respective arcs and zero on the remaining arcs. For each node $v \in V-\{s\}$, an initial label $l$ equal to its shortest path 
distance from the sink is assigned. The label of the source $s$ will always be $n$. A function $l: V \rightarrow N$ is a label function satisfying $l(s)=n(=|V|), l(d)=0$ and $l(v) \leq l(w)+1$ for every residual arc $e=(v, w) \in E_{f}$. Whenever the algorithm finds an active node $v$ (i.e. $e_{v}>0$ ) and if there is an arc in $N_{f}$ from node $v$ to node $w$ such that $l(v)=l(w)+1$, then $v$ pushes some excess less or equal to $c_{e=(v, w)}$ to $w$. If $l(w) \geq l(v) \forall w \in V$, label of $v$ is to be increased. We increase the label of an active node $v$ as $l(v):=1+\min \left\{l(w):(v, w) \in N_{f}\right\}$. If node $v$ satisfies $0 \leq l(v)<n$ then there is possibility of getting paths from $v$ to $d$. In other word, if $l(v) \geq l(s)$, the sink is not reachable from $v$ in $N_{f}$ and starts to send the flow back towards the source. The algorithm repeatedly performs these operations whenever applicable. At the termination, the algorithm gives a preflow with $e_{v}=0 \forall v \in V-\{s, d\}$. That is, a valid ordinary flow in the network. This flow is the maximum flow in the original network $N$.

It is not wise always (at the time of evacuation for example) to force sending the overload flow (evacuees) to the source rather to keep them at a temporary shelter at the intermediate node. Khadka and Bhandari [16] and Bhandari and Khadka [2] have purposed a modification on the preflow-push algorithm with an efficient solution procedure for a static network and have extended the problem on dynamic network. The solution for later case is based on time expanded network which has been explained in Section 4 below. Their modification solves the maximum evacuation planning problem with holding capability of flow at temporary shelter in intermediate nodes for single source single sink network. They consider the temporary shelter $v^{\prime}$ for each intermediate node $v \in V$ with sufficient capacity to make model simpler. The modified procedure does not send the evacuees reaching once at intermediate nodes back again to the source, a dangerous place but pushes back to any intermediate nodes. For an active node $v$, the overload flow $o_{v}$ is held in the temporary shelter $v^{\prime}$ at node $v$ if the push operation and the relabel operation are not applicable and even if $l(v) \leq l(s)$ does not satisfy after relabeling it.

\section{Maximum Dynamic Flow Problem}

The maximum flow problem seeks a feasible solution that sends the maximum amount of flow from a source to a sink with a maximum bound on the amount on each arc. A solution procedure to the problem with single-source-single-sink network $N=\left(V, E, c_{e}, \tau_{e}, s, d, T\right)$ with holding capability of the flow at intermediate nodes has been proposed in [16] and [2]. The procedure is based on the time-expanded network $N^{T}=\left(V^{T}, E^{T}, c_{e}, s\right)$ with

$$
\begin{gathered}
V^{T}=\{v(\theta): v \in V-\{s\} ; \theta \in\{0,1, \ldots, T\}\} \cup\{s\} \text { and } \\
E^{T}=\left\{\left(v(\theta), \quad w\left(\theta+\tau_{(v, w)}\right)\right): v \neq w ; v, w \in V-\{s\} ; \theta \in\left\{0,1, \ldots, T-\tau_{(v, w)}\right\}\right\} \cup \\
\left\{\left(s, w\left(\theta+\tau_{(s, w)}\right) ; \theta \in\left\{0,1, \ldots, T-\tau_{(s, w)}\right\}\right\} .\right.
\end{gathered}
$$

The limitation associated to this procedure is that it leads to a pseudo-polynomial time complexity since it strongly depends on $T$. To overcome the later limitation, we formulate the 
maximum dynamic flow problem as a typical minimum cost flow problem by interpreting the transit times as costs per unit flow on arcs. This minimum cost flow is decomposed into chain flows and apply temporal repetition over the given time horizon $T$ to find the maximum dynamic flow.

Consider a network $N=\left(V, E, c_{e}, a_{e}\right)$ where $a_{e}$ is the cost needed for unit flow to transship along arc $e \in E$. We also assign to each node $v$ a number $b_{v}$ representing its supply/demand. If $b_{v}>0$, node $v$ is a surplus node (a source); if $b_{v}<0$ node $v$ is a demand node (a sink); and if $b_{v}=0$, node $v$ is a transshipment node. Then the minimum cost flow problem asks to find a set of arc flows $f(e)$ that minimize a linear cost function

$$
\sum_{e \in E} a_{e} f(e)
$$

satisfying capacity constraint (1) with static setting and the mass imbalance constraint as

$$
\sum_{e \in \delta^{+}(v)} f(e)-\sum_{e \in \delta^{-}(v)} f(e) \geq b_{v} \forall v \in V .
$$

Now, we solve a minimum cost flow problem on the underlying static network. In the maximum dynamic flow problem we want to maximize the flow from the source to the sink within time horizon $T$. Thus, we need to model the time horizon $T$ in the minimum cost flow problem to be allowed to transfer the results of this problem to maximum dynamic flow problem. The solution procedure of the problem is basically based on the pre-flow push algorithm in [11] with necessary modification.

Instead of the label function representing the height as described in Section 3, we define another label function representing the node number $\pi: V \rightarrow Z^{+}$with $\pi_{s}=0, \pi_{d}$ at most $T+1$ and $\pi_{v}$ for $v \in V-\{s, d\}$ less than $\pi_{d}$. If $l(v)=l(w)+1$ for $e=(v, w) \in N_{f}$ together with $\pi_{v}+\tau_{(v, w)}=\pi_{w}$ for $f_{e=(v, w)}<c_{e=(v, w)}$ and $\pi_{w}+\tau_{(w, v)}=\pi_{v}$ for $f_{e=(v, w)}>0, \min \left\{e_{v}, c_{e}\right\}$, $e_{v}, c_{e}>0$ unit of flow is pushed from $v$ to $w$. The height of node $v$ is relabeled to be $l(v):=$ $1+\min \left\{l(w):(v, w) \in N_{f} ; w \neq s\right\}$ if $l(v) \leq l(w)$ with $e=(v, w) \in N_{f}$ and $e_{-} v>0$. And, the node number is relabeled as $\pi_{w}:=\pi_{w}+1$ for each $w \in N_{u}$ subset of $N_{f}$, the set of all inactive nodes not reachable from $v$ with respect to the arc cost. The overload flow $o_{v}$ is held in the temporary shelter $v^{\prime}$ of the node $v$ even if $l(v) \leq l(s)$ cannot be satisfied after relabeling. These operations are used to find the minimum cost flow $f_{m}$ on the $N=\left(V, E, c_{e}, a_{e}\right)$ by assuming an extra node as the source that connects to the original source node with infinite capacity and zero cost.

The procedure we described above seems to run faster than the procedure depending on timeexpanded network. In particular, for $C$ being the maximum of the cost over arcs on the networks, it terminates after $O\left(n^{2}\right)$ relabel operations, $O\left(n^{2} C\right)$ node number increasing operations, $O\left(n^{2} m\right)$ push operations and $O\left(n^{2}\right)$ hold operations. 
To solve the maximum evacuation planning problem on a dynamic network $N=\left(V, E, c_{e}, \tau_{e}\right.$, $s, d, T$ ) with hold of flow on intermediate nodes, we first compute the minimal cost flow as described above and decompose it into $s-d$ chain flows by using Routine 2 of Ford and Fulkerson [8]. We identify the nodes $v \in V-\{s, d\}$ with $o_{v}>0$ and decompose them also into $s-v$ chain flows. We remove all the arcs not belonging to any chain. We push as much flow as possible repeatedly from the source $s$ along each chain until the time step $T-\tau_{(s, v)},(s, v)$ being the first arc of each chain, and hold the overload flow at temporary shelter in the nodes along the chain. Resulting flow now is a maximum dynamic flow with hold of evacuees at temporary shelters.

Applying the procedure described above for the evacuation network given in Fig. 1 for time horizon $T=5$, we get the maximum dynamic flow of value 12 . Moreover, the procedure holds overload flow of 5,4 and 2 units at $p^{\prime}, q^{\prime}$, and $r^{\prime}$ respectively at time 5,1 and 4 units at $p^{\prime}$ and $q^{\prime}$ respectively at time 4 and 1 unit at $p^{\prime}$ at time 3 and 2 both.

\section{Concluding Remark}

This paper proposes a new and efficient solution technique that solves the maximum flow problem on single source single sink dynamic network with constant and integral transit times. The technique is based on non-conserving flow model introduced by Goldberg and Tarjan [11]. This paper also highlights the suitability of this problem on evacuation planning problem. More exact solution procedures for the proposed problem and model with its solution procedure in continuous time setting are the topics of immediate interest. Quickest flow problem and earliest arrival flow problem with non-conserving flow model would also be the future research works related to the topic.

Acknowledgements: The first author would like to thank University Grants Commission, Nepal for the PhD Fellowship award, 2016.

\section{References}

[1] Baumann N (2007), Evacuation by Earliest Arrival Flows. PhD Thesis, Department of Mathematics, University of Dortmund, Germany.

[2] Bhandari PP and Khadka SR (2017), Efficient Solution Approach to Maximum Flow Evacuation Planning Problem without Flow Conservation Aspect. Journal of Institute of Engineering, (Accepted).

[3] Burkard R, Dlaska K and Klinz B (1993), The Quickest Flow Problem. Mathematical Methods of Operations Research, 37(1) : 31-58.

[4] Dhamala TN and Pyakurel U (2013), Earliest Arrival Contraflow Problem on Series Parallel Graphs. International Journal of Operations Research, 10(1) : 1-13.

[5] Dinic EA (1970), Algorithm for Solution of a Problem of Maximum Flow in Networks with Power Estimation. Sov. Math. Dokl., 11: 1277-1280.

[6] Edmonds J and Karp RM (1972), Theoretical Improvements in Algorithmic Efficiency for Network Flow Problems. J. ACM, 19(2) : 248-264. 
[7] Ford LR and Fulkerson DR (1956), Maximal Flow through a Network. Can. J. Math., 8: 399404.

[8] Ford LR and Fulkerson DR (1958), Constructing Maximal Dynamic Flows from Static Networks. Operations Research, 6(3) : 419-433.

[9] Ford LR and Fulkerson DR (1962), Flows in Networks. Princeton University Press.

[10] Gale D (1959), Transient Flows in Networks. Michigan Mathematical Journal, 6 : 59-63.

[11] Goldberg AV and Tarjan RE (1988), A New Approach to the Maximum-Flow Problem. Journal of ACM, 35(4) : 921-940.

[12] Hoppe B (1995), Efficient Dynamic Network Flow Algorithms. PhD Thesis, Cornell University.

[13] Hoppe B and Tardos E (1994), Polynomial Time Algorithms for Some Evacuation Problems. Procc. of 5th Ann. ACM-SIAM Symp. on Discrete Algorithms, 433-441.

[14] Karzanov AV (1974), Determining a Maximal Flow in a Network by the Method of Preflows. Doklady Akademii Nauk SSSR, 215: 49-52, in Russian. (English translation in Soviet Math. Dokl, 15(2) : 434-437.

[15] Khadka SR and Bhandari PP (2017), Dynamic Network Contraflow Evacuation Planning Problem with Continuous Time Approach. International Journal of Operations Research, 14(1) : 27-34.

[16] Khadka SR and Bhandari PP (2017), An Efficient Solution Approach to Non-Conservation Maximum Flow Evacuation Planning Problem. Proceedings on SKIMA 2017, Colombo, 333335.

[17] Minieka E (1973), Maximal Lexicographic and Dynamic Network Flows. Operations Research, 21: 517-527.

[18] Pyakurel U and Dhamala TN (2015), Models and Algorithm on Contraflow Evacuation Planning Network Problems. International Journal of Operations Research, 12(2) : 36-46.

[19] Pyakurel U and Dhamala TN (2016), Continuous Dynamic Contraflow Approach for Evacuation Planning. Annals of Operations Research, 1-26 DOI: 10.1007/s10479-016-2302-5.

[20] Pyakurel U, Hamacher HW and Dhamala TN (2014), Generalized Maximum Dynamic Contraflow in Lossy Network. IJORN, 3 : 27-44.

[21] Rebennack S, Arulselvan A, Elefteriadou L and Pardalos PM (2010), Complexity Analysis of Maximum Flow Problem with Arc Reversal. Journal of Combinatorial Optimization, 19: 200216.

[22] Ruzika S, Sperber H and Steiner M (2011), Earliest Arrival Flows on Series-Parallel Graphs. Networks, 10: 169-173.

[23] Steiner M (2009), A Survey on Earliest Arrival Flows and a Study of the Series-Parallel Case. Diploma Thesis, Department of Mathematics, University of Kaiserslautern, Germany.

[24] Wilkinson WL (1971), An Algorithm for Universal Maximal Dynamic Flows in a Network. Operations Research, 19: 1602-1612. 